
\title{
25 Research Suare \\ Environmental Enrichment Protects Offspring of Preeclamptic-Like Rat Model From Cognitive Decline
}

Huiqing Lu

Obstetrics and Gynecology Hospital of Fudan University https://orcid.org/0000-0002-6636-0338

Lili Gong

Obstetrics and Gynecology Hospital of Fudan University

Huangfang Xu

Obstetrics and Gynecology Hospital of Fudan University

Qiongjie Zhou

Obstetrics and Gynecology Hospital of Fudan University

Huanqiang Zhao

Obstetrics and Gynecology Hospital of Fudan University

Suwen Wu

Obstetrics and Gynecology Hospital of Fudan University

Rong Hu ( $\nabla$ hurongwy@sina.com )

Fudan University

Xiaotian Li

Obstetrics and Gynecology Hospital of Fudan University

\section{Research}

Keywords: Preeclampsia, Cognition, Environment Enrichment, Neurogenesis, Apoptosis, Inflammation.

Posted Date: May 24th, 2021

DOl: https://doi.org/10.21203/rs.3.rs-504939/v1

License: (c) (1) This work is licensed under a Creative Commons Attribution 4.0 International License.

Read Full License 


\section{Abstract}

Background: Preeclampsia affects $5-8 \%$ of all pregnancies and contributes to adverse pregnancy and birth outcome. In addition to short-term effects of preeclampsia, it can exert long-term adverse effects on offspring. Numerous studies has demonstrated that offspring of preeclamptic women exhibited cognitive deficit from childhood to old age. However, effective ways to improving their cognitive ability remains to be investigated. The aim of this study was to explore whether Environmental Enrichment in early life could restore cognitive ability of offspring of preeclampsia rat model, as well as cellular and molecular mechanism by which EE improve cognitive ability.

Methods: L-NAME was used to establish preeclampsia rat model. The spatial learning and memory ability of 56-day-old offspring was evaluated by Morris Water Maze. Immunofluorescence was performed to evaluate cell proliferation and apoptosis in the DG region of hippocampus. qRT-PCR was performed to examine the expression level of neurogenesis-associated genes and inflammatory cytokines. Enzymelinked immune absorbent assay was performed to evaluate the concentration of Vascular endothelial growth factor (VEGF) and inflammatory cytokines in the hippocampus.

Results: The administration of L-NAME led to increased systolic blood pressure and urine protein level of pregnant rat. Offspring in L-NAME group exhibited impaired spatial learning ability and memory. The hippocampus neurogenesis and synaptic plasticity was impaired in offspring of L-NAME group. Furthermore, the cell apoptosis in hippocampus was increased in L-NAME group. The hippocampus was skewed to a pro-inflammatory profile, reflected by increased inflammatory cytokines level. While EE improved cognitive ability of offspring in L-NAME group and resulted in increased hippocampal neurogenesis and synaptic proteins expression level, decreased apoptosis level and inflammatory cytokines.

Conclusions: Environment Enrichment resolves cognitive impairment in offspring of preeclampsia rat model by improving hippocampal neurogenesis and synaptic plasticity, normalizing the apoptosis level and the inflammation balance.

\section{Background}

Preeclampsia has recently received increasing attention due to its long-term adverse effects on offspring. Preeclampsia (PE), a pregnancy-specific disorder, is characterized by de novo hypertension after 20th gestational week accompanied by associated end organ damage ${ }^{1}$. This disease affects $5-8 \%$ of all pregnancies worldwide, which is a major cause of maternal and neonatal morbidity and mortality, such as premature birth and intrauterine growth restriction. Besides these short-term adverse effects, long-term adverse effects of preeclampsia on offspring have been reported, including cardiovascular, metabolic and neurological disorders ${ }^{2}$. Nonetheless, the ways to improve the long-term outcomes remain to be investigated. 
In recent years, the impairment in neurological system of offspring exposed to preeclampsia has raised people's awareness. Growing evidence suggests that intrauterine exposure to preeclampsia can impair the cognition of offspring. Epidemiological studies revealed the association between PE and impaired cognition from childhood to old age. In one report, 7 to 10 year old children of preeclampsia women had an impairment in working memory and visuaspatial processing ${ }^{3}$. Ehrenstein et al. discovered that 18 to 19 year-old offspring exposed to preeclampsia were at increased risk of low cognitive function which was defined as $I Q<85^{4}$. Moreover, Tuovinen et al revealed that offspring aged 69.3 years who were born after preeclampsia were at increased risk of having impairment in memory and cognition compared with normotensive pregnancy ${ }^{5}$. Moreover, animal studies validated the causal relationship between preeclampsia and offspring cognitive impairment. Researchers found impaired brain structure and cognitive decline in offspring of preeclamptic-like rat model induced by L-NAME, a nitric oxide synthase inhibitor $^{6}$. These neurological changes may be attributed to neuro-inflammation and disrupted neurological function such as neurogenesis. However, there is no effective way to optimize offspring's cognition who were born after preeclampsia. Therefore, exploring a way to improve cognition of offspring is of great clinical significance.

Environment enrichment (EE) is the addition of physical, somatosensory and social stimulus to animals environment, including running wheels, toys, nesting materials, tubes and larger group housing ${ }^{7}$. It is reported that EE can lead to enhancements in hippocampal cognition and neuroplasticity and alleviate hippocampal cognitive deficits associated with neurodegenerative disease and aging ${ }^{8,9}$. We are interested in whether these changes which successfully enhance cognition have similar effects on offspring with cognitive decline who were born after preeclampsia rats. In the present study, we explored the effects of environmental enrichment on cognition alteration of offspring of preeclamptic rat model and the mechanism through which EE affected the cognitive ability.

\section{Methods And Materials}

\section{Animals}

For this study, 10 female and 5 male Sprague-Dawley rats, 8 weeks old, were purchased from company. All animals were habituated for 1 week to the housing room, kept under controlled conditions, temperature of $21^{\circ} \mathrm{C}$, humidity of $50 \%$, a 12-h light/12-h dark cycle (lights on $8 \mathrm{am}$ ) and they had access to food and water ad libitum. All procedures were approved by the Animal Care and Use Committee of the University of Fudan. All efforts were made to minimize animal suffering and reduce the number of animals used.

\section{Mating and the establishment of preeclampsia model}

At age of 9 weeks, rats were mated in groups of 2 females and 1 male. The vaginal plugs were carefully looked for in cages everyday around $7 \mathrm{am}$. When vaginal plugs were observed, female rats were considered pregnant and separated. This date was defined as gestational day (GD) 0 . To establish the 
preeclampsia animal model, L-NAME, an NO synthesis inhibitor, was chosen which was usually used to establish preeclampsia rat model. Briefly, pregnant dams either received L-NAME $(125 \mathrm{mg} / \mathrm{kg}$ subcutaneous, Sigma-Aldrich) or $\mathrm{NaCl}$ as vehicle from GD13 to GD21 (Figure 1). All solutions were freshly prepared everyday. The blood pressure and urine protein were detected to validate the establishment of preeclampsia model. The blood pressure was detected with BP-2000 Blood Pressure analyzer via a tail cuff before and after the administration of L-NAME. The protein level in urine was quantified using Bradford method (P0006C, Beyotime). A total of 18 male offspring were analyzed in this study. Animals were weaned at postnatal day 21.

\section{Experimental housing}

Between postnatal day (PND) 21 and 56, male offspring were housed in standard housing (SH) condition or enriched environment(EE). In standard housing condition, 3 littermates were kept together in one rat cage with sawdust bedding material. In enriched environment, 6 Rats were housed in a large cage $(100 \times 60 \times 90 \mathrm{~cm})$, which included a free-running wheel, two plastic tunnel, a raised platform, a stair case and various colored ball. To increase novelty, these objects were exchanged once a week during cage cleaning. These environmental stimuli and increased number of partners improved environmental complexity compared with SH. Group sizes were: $\mathrm{NaCl}$ SH: $n=6$, L-NAME SH: n=6, L-NAME EE: n=6.

\section{Morris Water Maze}

After 5 weeks of experimental housing, all animals were housed under standard housing conditions for one week before starting behavioral test. The cognitive ability was evaluated by Morris Water Maze, which was widely used for testing the animals' spatial learning ability and memory. This test involves two stages, the spatial learning stage and the memory test stage. In the spatial learning stage, a circular platform was placed at a specific location away from the edge of the pool. The platform was submerged $1.5 \mathrm{~cm}$ below the water surface. The rats were trained in three trials per day. The animals were placed at a certain position and given $60 \mathrm{~s}$ to find the platform. If the animal could not find the platform, it was guided to the platform and was allowed to stay on the platform for $20 \mathrm{~s}$. The training procedure lasted for 4 days.

In the memory test stage, the platform was removed and the trained animals were placed at a specific position in the pool to swim. The time they spent in each quadrant (target quadrant, left quadrant, opposite quadrant and right quadrant), the latency to the platform area and the frequency of reaching the platform area were recorded. All data from the water maze test were collected with a video camera fixed to the ceiling and connected to a computer and a video-tracking system (Noldus Information Technoloy, Holland).

\section{Sample preparation and immunofluorescence}

Experimental rats were anesthetized with chloral hydrate and perfused transcardially with cold phosphate-buffered saline (PBS), followed by $4 \%$ paraformaldehyde (PFA). The hippocampus was 
dissected out and fixed in $4 \%$ PFA at $4{ }^{\circ} \mathrm{C}$. Then, the tissues were sliced into $20-\mu \mathrm{m}$ coronal sections on glass slides. The immunofluorescence assays was to detect Brdu+ and TUNEL+ cells, which could indicate the neurogenesis and apoptotic cells in hippocampus, respectively. The offspring were injected with Brdu solution according to the procedure (Figure 1). To evaluate the apoptotic cells, the hippocampus sections were stained by using fluorometric terminal deoxynucleotidyl transferase deoxyuridine triphosphate (dUTP) nick end labeling (TUNEL, KGA7073, KeyGEN BioTECH) staining according to the manufacturer's instructions. Images were analyzed using confocal microscope.

\section{Quantitative Real-Time PCR}

Total hippocampus RNA was extracted by using TRIzol (Invitrogen, 15596026, Carlsbad, CA, USA). Complementary DNA (cDNA) synthesis was performed by using PrimeScript ${ }^{\text {TM }}$ RT reagent Kit with gDNA Eraser (RR047A Takara). cDNA was subjected to RT-PCR using Hieff UNICON Universal BLUE qPCR SYBR Master Mix (11184ES08, YEASEN) according to the manufacturer's instructions. The quantitative expression level of neurogenesis-related genes including Fgf, PTN, EP300, Creb, BNDF and NGF as well as inflammatory cytokines including IL-1 $\beta$, IL- 6 and TNF- $a$ were evaluated by qRT-PCR. The primer sequences were listed in Table 1.

\section{ELISA assay}

The ELISA assay was performed to determine the levels of VEGF and inflammatory factors in hippocampus of offspring. Briefly, after the samples were collected, these tissues were homogenized with normal saline and centrifuged at $2000 \mathrm{rpm}, 4^{\circ} \mathrm{C}$ for $20 \mathrm{~min}$. The supernatants were collected. The protein level of supernatants was quantified using Bradford method (P0006C, Beyotime). The concentrations of VEGF, IL-1 $\beta$, IL- 6 and TNF-a were assessed with a commercially available enzyme-linked immunosorbent assay (ELISA) kit: Rat VEGF-A ELISA (RayBiotech, USA), Rat IL-1 beta ELISA (RayBiotech, USA), Rat IL-6 ELISA (RayBiotech, USA), Rat TNF-alpha ELISA (RayBiotech, USA) according to the manufacturer's instructions.

\section{Statistical analysis}

All statistical analyses were conducted in GraphPad Prism 7.0 software. Data were presented as mean value and standard error of the mean (SEM). Statistical significance was determined by Student's $t$ test or one-way ANOVA. A probability level of $\mathrm{P}<0.05$ was considered statistically significant.

\section{Results}

\section{The establishment of preeclampsia-like rat models}

To confirm whether the preeclampsia animal model was successfully established, blood pressure and $24 \mathrm{~h}$ urine protein of pregnant rat was examined. We found the administration of L-NAME led to an increase in systolic blood pressure. Before the administration of L-NAME, the average systolic blood pressure was $126.20 \mathrm{mmHg}$. However, the average SBP was as high as $146.53 \mathrm{mmHg}$ after using it 
(Figure 2a, $p<0.001$ ). Meanwhile, it also led to an significant increase in urine protein level in pregnant rat (Figure $2 b, p<0.001$ ). In addition, the body weight of neonates in the preeclampsia group was significantly lower $(5.76 \pm 0.63 \mathrm{~g})$ than that of the control group $(7.72 \pm 0.85 \mathrm{~g})$ (Figure $2 \mathrm{c}, \mathrm{p}<0.001)$. The number of neonates in preeclampsia group was also decreased compared with the control group (Figure $2 d, p<0.05$ ). Furthermore, there was limb defect in some neonates of preeclampsia group (Figure $2 e, p>0.05$ ).

\section{Improvement in learning and memory ability in the offspring of L-NAME group after EE}

To analyze the learning ability and memory of the offspring, Morris water maze was used. In both stage, the performance of offspring in EE group was indistinguishable from that of offspring in control group- in sharp contrast to offspring in L-NAME group.

In the training stage, the latency to platform was progressively decreased in control group (Figure 3a), which indicated that offspring learned the task from day 1 to day4. However, latency to platform in LNAME group decreased much slower, less efficient and longer than that in control group (Figure 3a, $p<0.005)$. While EE could largely restore the learning ability deficient, reflected by the decrease in latency to platform (Figure 3a, $\mathrm{p}<0.005$ ). There is no significant difference in swimming speed among 3 groups (Figure $3 b, p>0.05$ ). The performance in the training stage revealed that offspring in L-NAME group had impaired learning abilities and EE could improve it.

At the memory test stage, the results showed that offspring in L-NAME group displayed much higher latency to training platform area, compared with control group(Figure $4 a, p<0.005$ ). A shorter swimming distance in target quadrant was found L-NAME group (Figure $4 b, p<0.005$ ). They also spent significantly less time in platform quadrant than offspring in control group (Figure $4 c, p<0.005$ ). Additionally, we found the frequency of crossing platform in L-NAME group was much lower than that in control group (Figure $4 d, p<0.001)$. All these changes could be reversed by EE. Therefore, we have demonstrated that EE had protected offspring of PE from impaired memory.

\section{Increased neurogenesis in hippocampus of offspring of preeclampsia after EE}

To reveal cellular and molecular mechanism through which EE improves brain function in the offspring of preeclamptic rat, we investigated the hippocampal neurogenesis, which was reported to be associated with spatial learning ability and memory. Immunofluorescence (IF) results showed that numbers of BrdU+ cells was significantly decreased in L-NAME group, while it was increased in EE group (Figure 5a,5b, $p<0.005)$, indicating that decreased neurogenesis was resolved by EE. Next, qRT-PCR was used to test the expression level of adult hippocampal neurogenesis - associated genes including Fgf, PTN, EP300, Creb, BNDF and NGF. However, there were no significant difference in expression levels of these genes among three groups (Figure $5 c, p>0.05$ ). We then tested whether there was changes in VEGF concentration. VEGF was reported to promote neurogenesis in adult brain possibly through enhancement in vascular niche. The result showed a significant reduction of VEGF in L-NAME group when compared with young group. However, EE restored hippocampal VEGF to a level similar to those of control group (Figure $5 d, p<0.05$ ). 
Thus, EE prevent hippocampal VEGF decrease in L-NAME offspring, possibly underlying its positive effect on neurogenesis.

\section{Increased synaptic plasticity in hippocampus of the offspring of the L-NAME group after EE}

To evaluate the synaptic plasticity in hippocampus, we assessed the mRNA expression level of pre- and postsynaptic proteins synapsin, PSD95 and SNAP25. We found decreased expression levels of synapsin (Figure 6a, Control vs L-NAME, $\mathrm{p}<0.005$, L-NAME vs EE, $\mathrm{p}<0.05$ ) and PSD95 in L-NAME group (Figure 6b, $p<0.05)$ compared with control group. However, the expression level of these synaptic-related protein was restored by EE. No significant difference in SNAP25 level was observed among these groups (Figure 6c, $\mathrm{p}>0.05)$.

\section{Reduced apoptosis in hippocampus of the offspring of the L-NAME group after EE}

Neural apoptosis in DG region of hippocampus, measured by the staining of TUNEL+ nuclei, was significantly different among three groups (Figure 7a). The number of TUNEL+ cells were increased in LNAME group when compared with control group as well as EE group. No significant difference existed between control and EE group (Figure $7 b, p<0.001$ ). The results indicated that EE could alleviated neural apoptosis in L-NAME group.

\section{Reduced inflammation in hippocampus of offspring of preeclampsia after EE}

Considering the strong association between cognitive impairment and inflammation, we examine the inflammatory cytokines in hippocampus. Both qRT-PCR and ELISA results revealed different inflammatory profiles in these groups. The results showed both mRNA and protein levels of proinflammatory cytokines including IL-1 $\beta$ (Figure 8a, Control vs L-NAME, $p<0.005$, L-NAME vs EE, $p<0.05$. Figure $8 b$, Control vs L-NAME, $p<0.001$, L-NAME vs EE, $p<0.05$ ) and IL- 6 were increased in L-NAME group compared with control and EE group (Figure 8c, Control vs L-NAME, $p<0.001$, L-NAME vs EE, $p<0.005$. Figure $8 d$, Control vs L-NAME, $p<0.005$, L-NAME vs EE, $p<0.05$ ). Nonetheless, no significant difference was found in TNF-a level among three groups (Figure $8 e, f, p>0.05$ ). These results suggested that EE could reverse excessive hippocampal inflammation in L-NAME offspring.

\section{Discussion}

In this study, we examined EE intervention in early life for the prevention of the preeclampsia-related cognitive decline in adolescent offspring in L-NAME rat model. Previous epidemiological studies has demonstrated that maternal preeclampsia are strongly associated with a poorer cognitive performance of their children. In consistent with this, we found that the administration of L-NAME to pregnant rats induced cognitive deficits in offspring. Offspring in L-NAME group exhibited pathological changes including impaired neurogenesis and synaptic plasticity, increased neural apoptosis and levels of inflammatory cytokines in hippocampus compared with their counterparts in Control group. Notably, rearing offspring in L-NAME group in an enriched environment for five weeks prevented hippocampus- 
dependent learning ability and spatial memory decline as well as pathological changes in hippocampus. Altogether, these results indicated that $\mathrm{EE}$ might be effective in restoring cognitive changes predisposed by adverse intrauterine environment.

We used L-NAME rat model of preeclampsia, in which L-NAME (an inhibitor of NOS ) was administrated to pregnant rat during Gestational Day 13 to 21 . This model is effective in exploring preventive strategies for cognitive decline of offspring who were born after preeclampsia due to its ability to recapitulate the clinical features of preeclampsia including increased blood pressure and urine protein. In preeclampsia women, the NO production is reduced. L-NAME rat model could mimic NO deficiency. Moreover, since Gestational Day 13 to 21 is a critical stage for brain development, this model is effective in studying the neurodevelopment of preeclampsia offspring. A previous study from our laboratory reported the spatial learning ability and learning ability decline as well as impaired neurological development in adolescent offspring of L-NAME rat, which strengthening the effectiveness of this model.

Studies exploring ways to improving cognitive ability of offspring of preeclampsia were few and mainly focused on gestational diet interventions. To our knowledge, the present study represents the first examination of whether EE could protect offspring of preeclampsia against cognitive decline and thus provides novel insights for early intervention. We showed that $E E$ in early life is sufficient to prevent cognitive deficits in adolescent offspring from L-NAME group. There are various ways to provide enriched environment, thus, the protocols lack consistency. However, the most common procedure includes rearing the rats in a large cage and providing them with novel subjects and social contact for at least 30 days immediately after weaning. The procedure provides key factors of EE including social contacts, novelty and exercise, all of which have been reported to be rewarding. The offspring were weaned at postnatal day 21 . Therefore, they were reared in enriched environment from postnatal day 21 to day 56 , which lasted for five weeks.

In this study, Morris Water Maze was used to assess hippocampus-dependent cognitive function of offspring. Our data showed that offspring in L-NAME group exhibited a clear decline in spatial learning ability, reflected by increased "Latency to platform ", which was prevented by EE. With regard to spatial memory, L-NAME induced spatial memory decline indicated by shorter swimming distance, less time in target quadrant and lower "frequency of crossing platform" in test stage. The impaired spatial memory was also revolved by EE. These results showed an impaired hippocampus-dependent learning ability and spatial memory in L-NAME group. However, after 5 weeks of EE intervention, the performance of offspring in Morris water maze was dramatically improved.

In an attempt to discern the biological underpinnings of the observed cognitive changes, we focused on structural and molecular plasticity of hippocampus. The results showed that EE could improve neurogenesis, attenuate neural apoptosis, improve synaptic plasticity. Moreover, EE normalize the inflammation balance in hippocampus by decreasing the expression of pro-inflammatory cytokines IL-1 $\beta$ and IL-6. 
Hippocampus is a key structure involved in learning and memory. Adult hippocampus could continuously generate new neurons that are integrated to hippocampal circuits. These newly generated neurons are thought to play an important role in hippocampal-dependent spatial learning and memory (BruelJungerman et al., 2007; Shors et al., 2001). The process of hippocampal neurogenesis has been reported to be influenced by various factors including physiological conditions and environmental stimuli. Therefore, we investigate neurogenesis in DG where new neurons are added to mature circuit. Our study showed that the exposure to an adverse uterine environment exerted an negative effect on hippocampal neurogenesis, reflected by decreased number of Brdu + cells in DG region of offspring hippocampus, while five weeks of EE intervention in early life could restore this changes. This suggested that impaired cognitive function in L-NAME offspring may be associated with reduced neurogenesis, which is attenuated by EE.

Alterations in hippocampus growth factors might be functionally linked with neurogenesis changes. BDNF has been widely studied as the candidate mediating hippocampal neurogenesis changes induced by environmental stimuli. For instance, the deletion of TrkB (BDNF receptor) reduced the effects of exercise on adult neurogenesis. In addition, NGF impacts on the survival of neuronal progenitor cells. Amy et al. found that the cognitive decline with aging were associated with a reduction of NGF ${ }^{10}$. Moreover, FGF signaling pathways play a role in regulating neurogenesis. The deletion of FGF receptor genes could result in a dramatic loss of neurogenesis in the DG, while enhancing FGF receptor activity in neurogenic cells could increase their numbers ${ }^{11}$. VEGF is also reported to enhance neurogenesis ${ }^{12}$. Gao et al. reported reduced VEGF expression with aging might exert impact on the angiogenic niche within the DG and reduce neurogenesis ${ }^{13}$. VEGF could enhance the angiogenic niche in the subgranular zone(SGZ) of the DG. In support with these studies, blockade of VEGF could eliminate exercise-induced improvements in neurogenesis, indicating that VEGF may play a significant role in the stimulation of neurogenesis ${ }^{14}$. These studies indicated important roles of these growth factors in regulation of neurogenesis. Accompanying the changes in neurogenesis, we found decreased expression levels of VEGF, while there were no changes in BDNF, NGF, FGF and other neurogenesis-related genes expression levels. Interestingly, the reduction in VEGF were attenuated in EE group. Furthermore, increased neural apoptosis level was observed in L-NAME group, which was attenuated by EE. Therefore, the improved neurogenesis by EE may be partly attributed by increased expression of VEGF and inhibition of apoptosis.

Previous studies have provided substantial link between neuropsychological disorders and neuroinflammation. McAffose et al. demonstrated that elevated or prolonged exposure to inflammatory mediators could have detrimental effects on cognitive function. Moreover, growing evidence implicated dysregulated inflammation in the development of $A S D^{15}$. Masi et al. reported a number of cytokines were dysregulated in ASD and correlated with severity of ASD symptom ${ }^{16}$. Similar to this findings, in this study, we found increased inflammatory cytokines in preeclampsia offspring compared with normal pregnancy. The data in our study showed a pro-inflammatory phenotype in hippocampus of offspring in L-NAME group, reflected by increased levels of IL-1 $\beta$ and IL- 6 , which is attenuated by EE. IL- $1 \beta$ has been widely reported to have memory-modulating effects and increased in many neurodegenerative diseases and 
normal aging 17,18 , thus, the EE induced modulation of IL-1 expression could help preserve cognition function. Additionally, EE induced improvement in cognitive ability could be partly attributed to the attenuation of increase in IL-6, which could impact synaptic plasticity and neurodegeneration. Indeed, in this study, we found decreased expression level of pre- and postsynaptic proteins synapsin, PSD95 and SNAP25 in L-NAME group compared with control group, while EE could rescue this reduction. Notably, recent studies have demonstrated that neuroinflammation is a potent inhibitor of hippocampal neurogenesis. Thus, we speculated that reduced inflammation induced by EE attenuated cognitive impairment through improving hippocampal neurogenesis and synaptic plasticity.

\section{Conclusion}

In this study, we demonstrated that the administration of L-NAME during pregnancy could lead to an impairment in hippocampus-dependent learning ability and spatial memory, accompanied by pathological changes including decreased neurogensis and VEGF expression, lower levels of synaptic proteins, increased apoptosis as well as pro-inflammatory cytokines. Five weeks of EE in early life could improve learning ability and spatial memory and restore these changes.

\section{Abbreviations}

EE: Environmental enrichment, SH: Standard housing, BDNF: brain-derived neurotrophic factor, NGF: Nerve growth factor, VEGF: Vascular endothelial growth factor, L-NAME: N( $\omega)$-nitro-L-arginine methyl ester, PE: Preeclampsia, GD: Gestational day, PND: Postnatal day, ELISA: Enzyme-linked immunosorbent assay, PBS: Phosphate-buffered saline, PFA: Paraformaldehyde.

\section{Declarations}

\section{Acknowledgement}

We would like to thank the professor Hong Jin from Fudan University.

\section{Authors' contributions}

Xiaotian Li, Rong Hu and Huiqing Lu conceived the study, designed the experiments and wrote this manuscript. Huiqing Lu performed the experiments with help of Lili Gong, Huangfang Xu, Huanqiang Zhao and Suwen Wu. Qiongjie Zhou contributed to data analysis. All authors read and approved the final manuscript.

\section{Funding}

This study was supported by the National Natural Science Foundation of China(No.81571460).

\section{Availability of data and materials}


The data supporting the main findings and conclusions of this article are included within the article. All datasets and analyses used in the this study are available from the corresponding author on reasonable request.

\section{Ethics approval and consent to participate}

All procedures in this study were approved by the Animal Care and Use committee of the University of Fudan and in accordance with the animal care guidelines of the National Institute of Health.

\section{Consent for publication}

This manuscript has been approved for publication by all authors.

\section{Competing interests}

The authors declare that they have no competing interests.

\section{References}

1. Brown MA, Magee LA, Kenny LC et al. Hypertensive Disorders of Pregnancy: ISSHP Classification, Diagnosis, and Management Recommendations for International Practice. Hypertension. 2018,72(1):24-43

2. Lu HQ, Hu R. Lasting Effects of Intrauterine Exposure to Preeclampsia on Offspring and the Underlying Mechanism. AJP Rep. 2019,9(3):e275-e291

3. Ratsep MT, Hickman AF, Maser B et al. Impact of preeclampsia on cognitive function in the offspring. Behav Brain Res. 2016,302:175-81

4. Ehrenstein V, Rothman KJ, Pedersen L, Hatch EE, Sorensen HT. Pregnancy-associated hypertensive disorders and adult cognitive function among Danish conscripts. Am J Epidemiol. 2009,170(8):102531

5. Tuovinen S, Aalto-Viljakainen T, Eriksson JG et al. Maternal hypertensive disorders during pregnancy: adaptive functioning and psychiatric and psychological problems of the older offspring. BJOG. 2014,121(12):1482-91

6. Liu X, Zhao W, Liu H et al. Developmental and Functional Brain Impairment in Offspring from Preeclampsia-Like Rats. Mol Neurobiol. 2016,53(2):1009-1019

7. Ohline SM, Abraham WC. Environmental enrichment effects on synaptic and cellular physiology of hippocampal neurons. Neuropharmacology. 2019,145(Pt A):3-12

8. Grinan-Ferre C, Perez-Caceres D, Gutierrez-Zetina SM et al. Environmental Enrichment Improves Behavior, Cognition, and Brain Functional Markers in Young Senescence-Accelerated Prone Mice (SAMP8). Mol Neurobiol. 2016,53(4):2435-50

9. Wei Z, Meng X, El FR et al. Environmental enrichment prevents Abeta oligomer-induced synaptic dysfunction through mirna-132 and hdac3 signaling pathways. Neurobiol Dis. 2020,134:104617 
10. Birch AM, Kelly ÁM. Lifelong environmental enrichment in the absence of exercise protects the brain from age-related cognitive decline. Neuropharmacology. 2019,145(Pt A):59-74

11. Kang W, Hébert JM. FGF Signaling Is Necessary for Neurogenesis in Young Mice and Sufficient to Reverse Its Decline in Old Mice. J Neurosci. 2015,35(28):10217-23

12. Fabel K, Fabel K, Tam B et al. VEGF is necessary for exercise-induced adult hippocampal neurogenesis. Eur J Neurosci. 2003,18(10):2803-12

13. Gao P, Shen F, Gabriel RA et al. Attenuation of brain response to vascular endothelial growth factormediated angiogenesis and neurogenesis in aged mice. Stroke. 2009,40(11):3596-600

14. Fabel K, Fabel K, Tam B et al. VEGF is necessary for exercise-induced adult hippocampal neurogenesis. Eur J Neurosci. 2003,18(10):2803-12

15. Matta SM, Hill-Yardin EL, Crack PJ. The influence of neuroinflammation in Autism Spectrum Disorder. Brain Behav Immun. 2019,79:75-90

16. Masi A, Glozier N, Dale R, Guastella AJ. The Immune System, Cytokines, and Biomarkers in Autism Spectrum Disorder. Neurosci Bull. 2017,33(2):194-204

17. Frank MG, Barrientos RM, Biedenkapp JC et al. mRNA up-regulation of MHC II and pivotal proinflammatory genes in normal brain aging. Neurobiol Aging. 2006,27(5):717-22

18. Lynch MA. Age-related neuroinflammatory changes negatively impact on neuronal function. Front Aging Neurosci. 2010,1:6

\section{Tables}

Table 1 is not available with this version.

\section{Figures}




\begin{tabular}{l|l|}
$\operatorname{Control}(n=6)$ & Five weeks of $S H$ \\
$\operatorname{L-NAME}(n=6)$ & Five weeks of $S H$ \\
$\operatorname{EE}(n=6)$ & Five weeks of EE \\
\hline
\end{tabular}

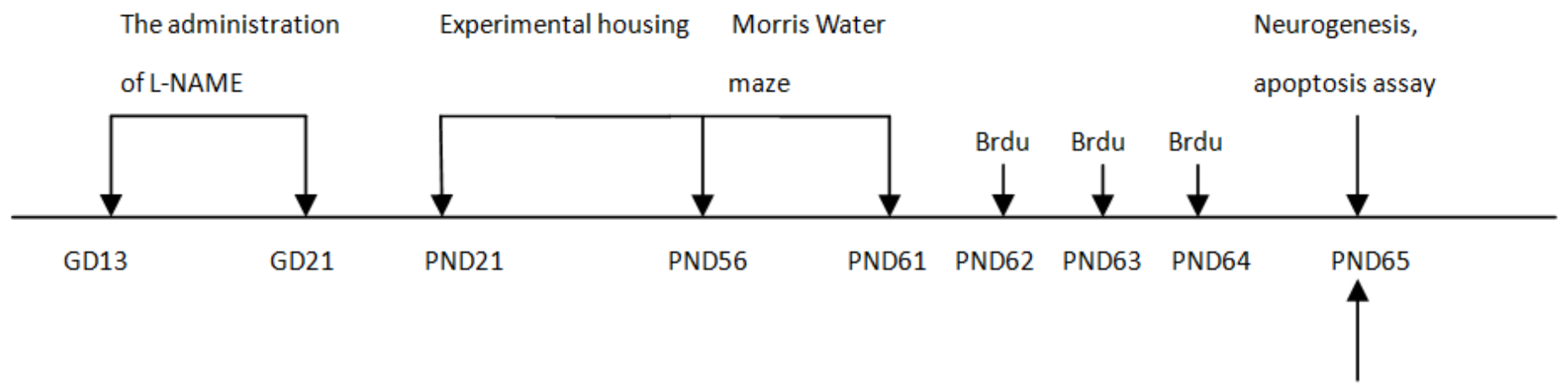

Synaptic plasticity and inflammatory cytokines assay

Figure 1

Experiment procedure and timeline 

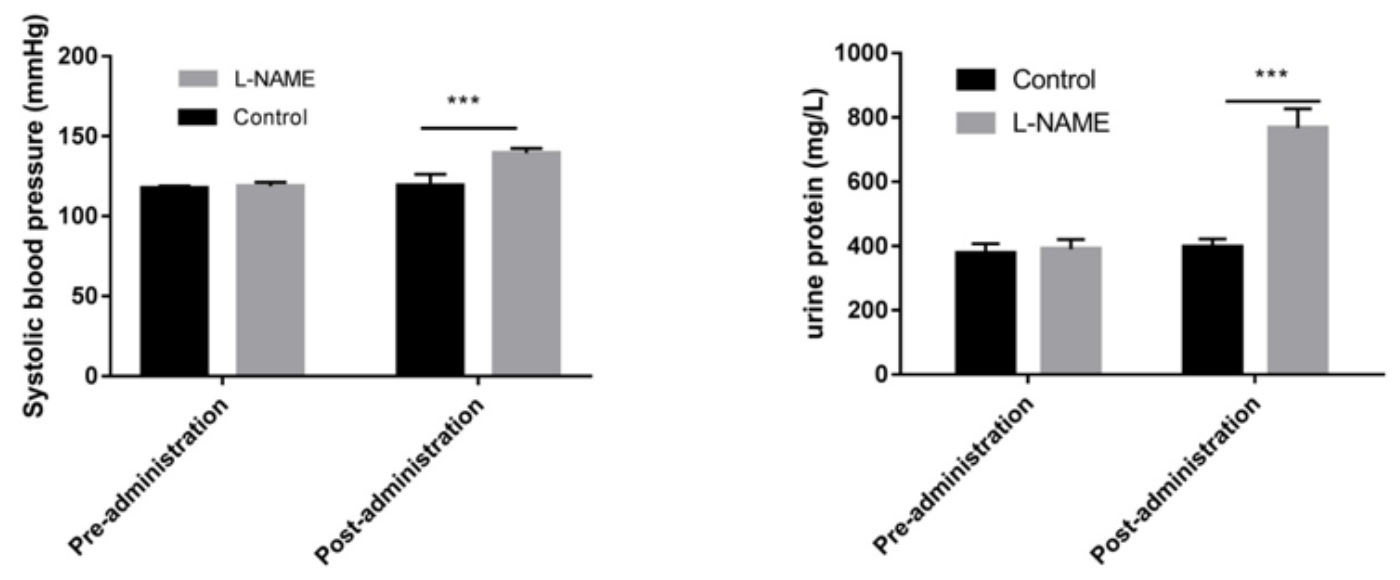

C

d
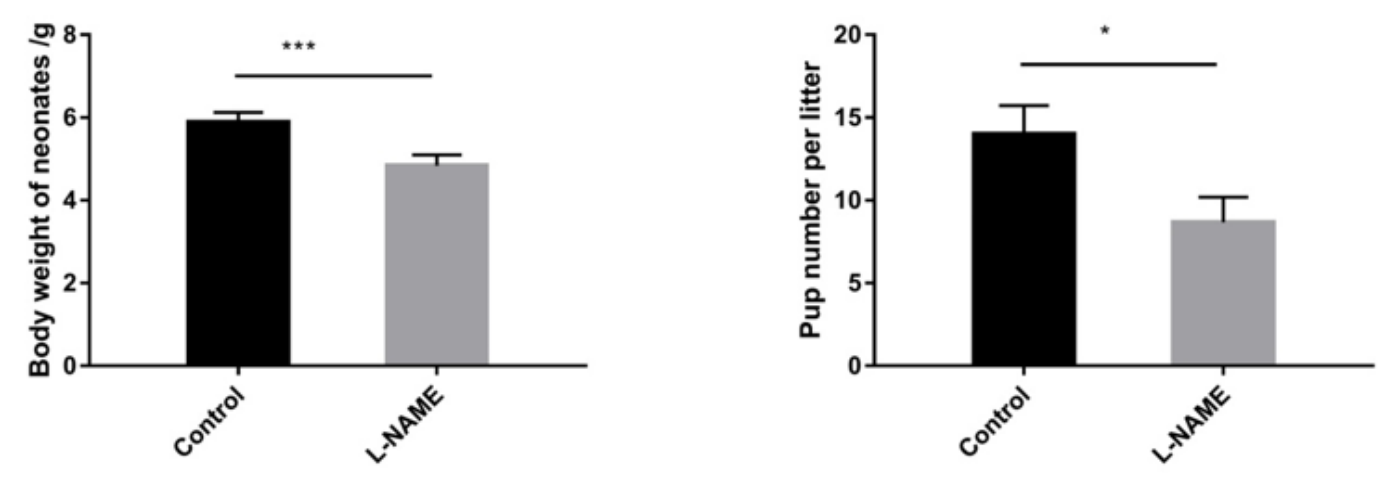

e

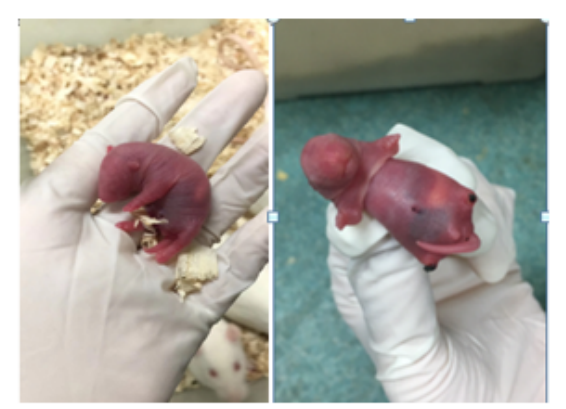

\section{Figure 2}

The establishment of preeclampsia-like model in rats. a Systolic blood pressure of pregnant rat in Control group and L-NAME group. b Urine protein levels of pregnant rat in Control and L-NAME group. c Body weight of pups at Postnatal day 1 in Control and L-NAME groups. $d$ The number of pups per litter in Control and L-NAME group. e Limb defects in PE group induced by L-NAME. Statistical analysis was performed using Student's t test. * $p<0.05$. *** $p<0.001$. 
a

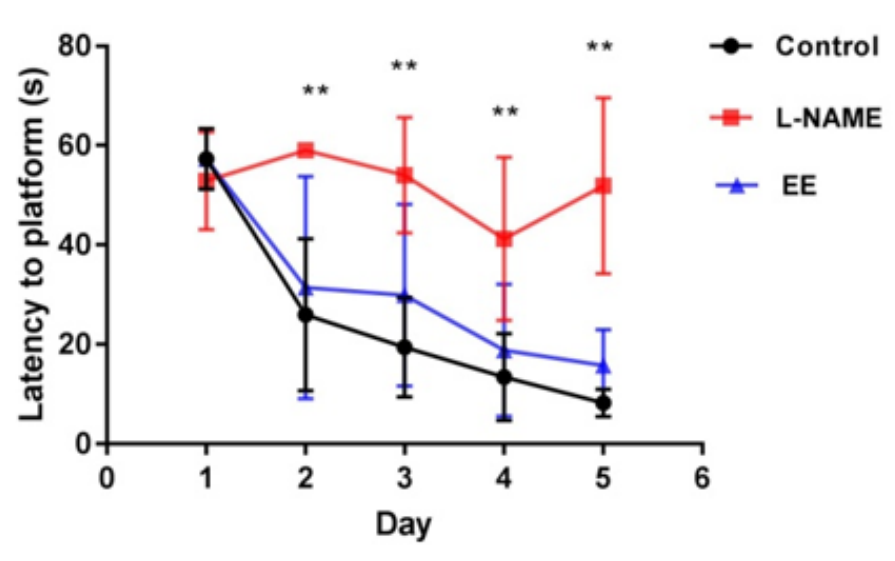

C
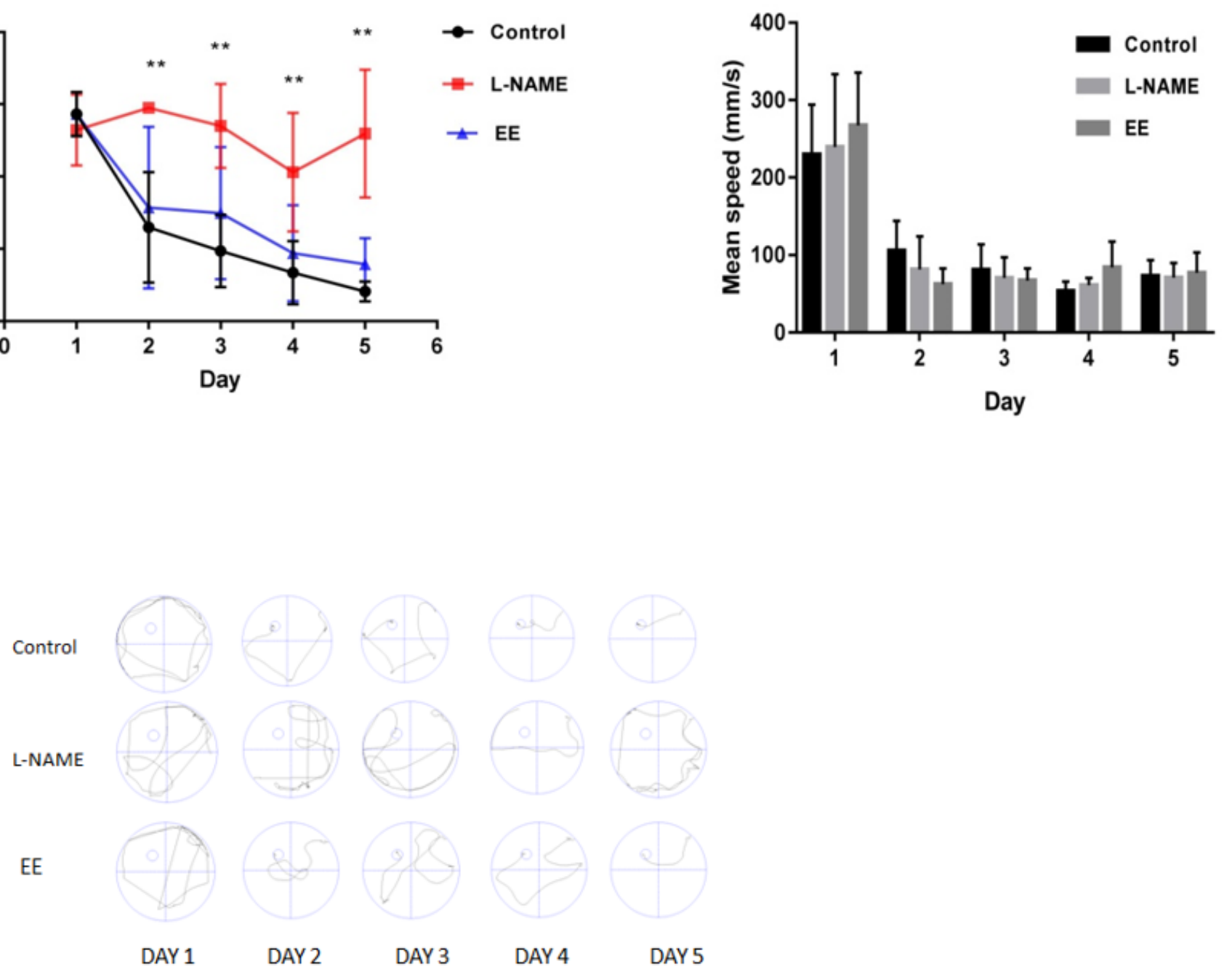

Figure 3

Effect of Environmental Enrichment on spatial learning ability. a Latency to platform of offspring in each group on training stage. b Mean swimming speed of each group on training stage. c Tracks of tested offspring in each group. Statistical analysis was performed using one-way ANOVA. ** $p<0.005$. 
a

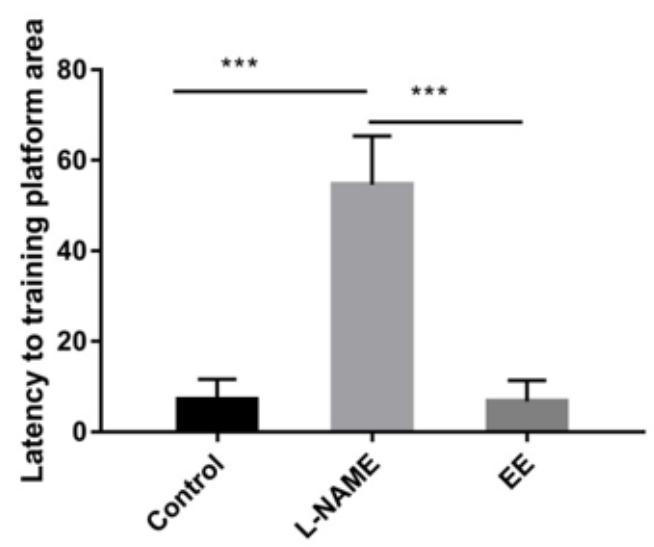

c

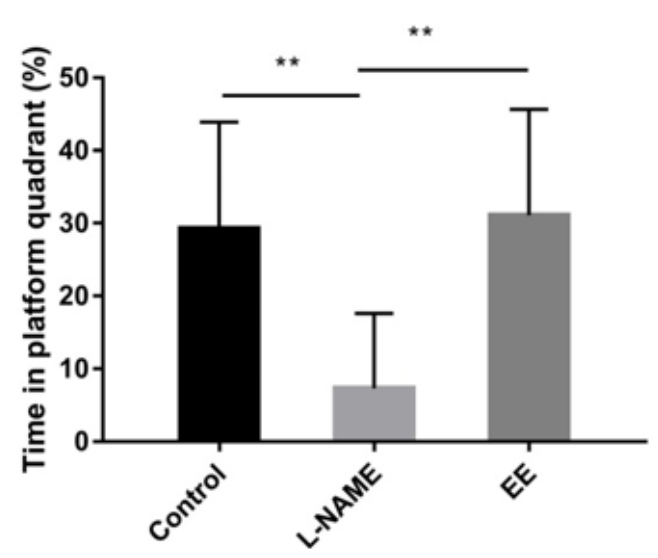

e

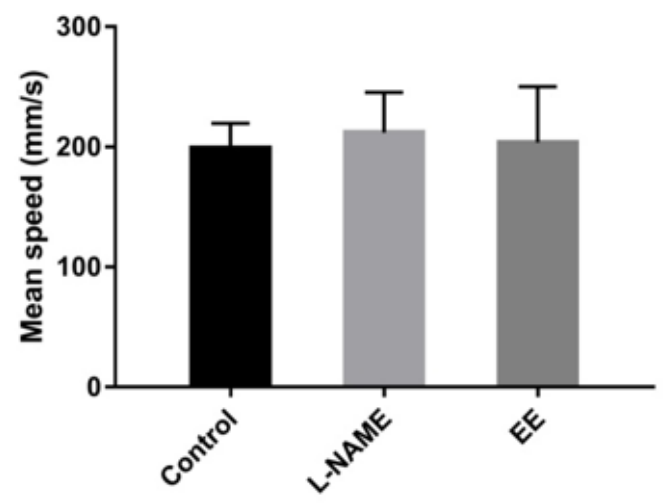

b

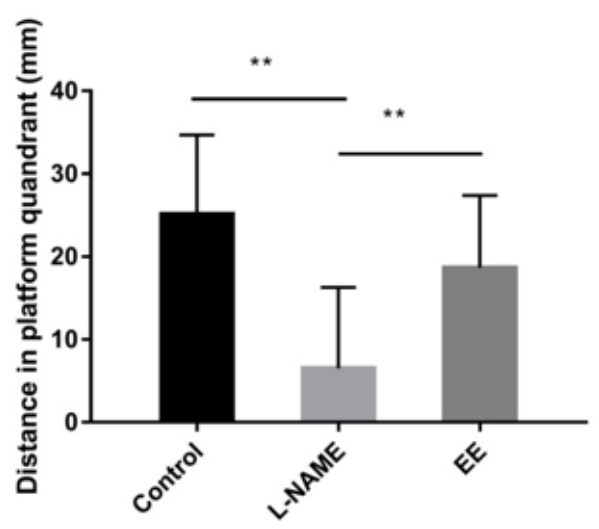

d

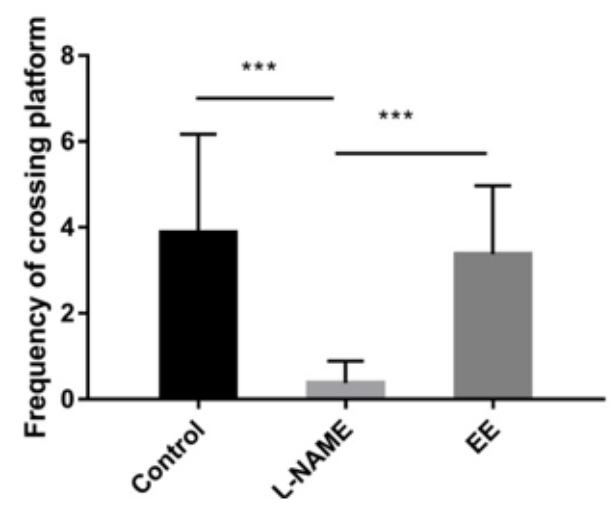

f

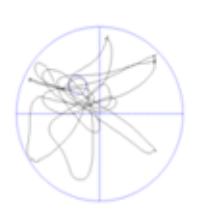

Control

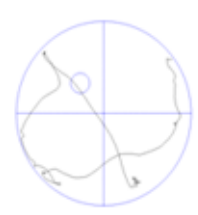

L-NAME

EE

\section{Figure 4}

Effect of Environmental Enrichment on memory. a Frequency of crossing the platform area of each group. b Swimming distance in platform quadrant of each group. c Time spent in platform quadrant of each group. $d$ Frequency of crossing platform quadrant in each group. e Mean swimming speed of each group. $f$ Typical behavior tracks. Statistical analysis was performed using one-way ANOVA. $* \star p<0.005$. $\star \star \star x<0.001$. 
a

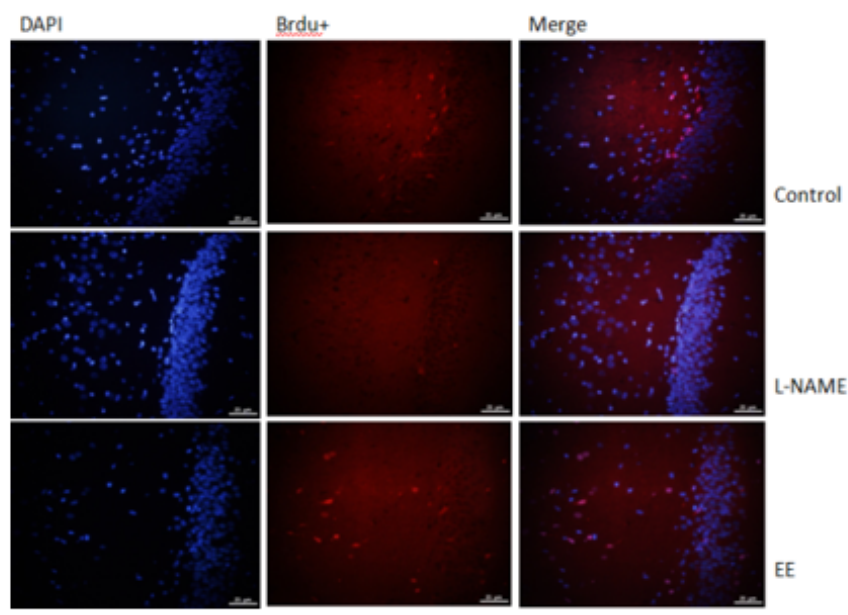

C

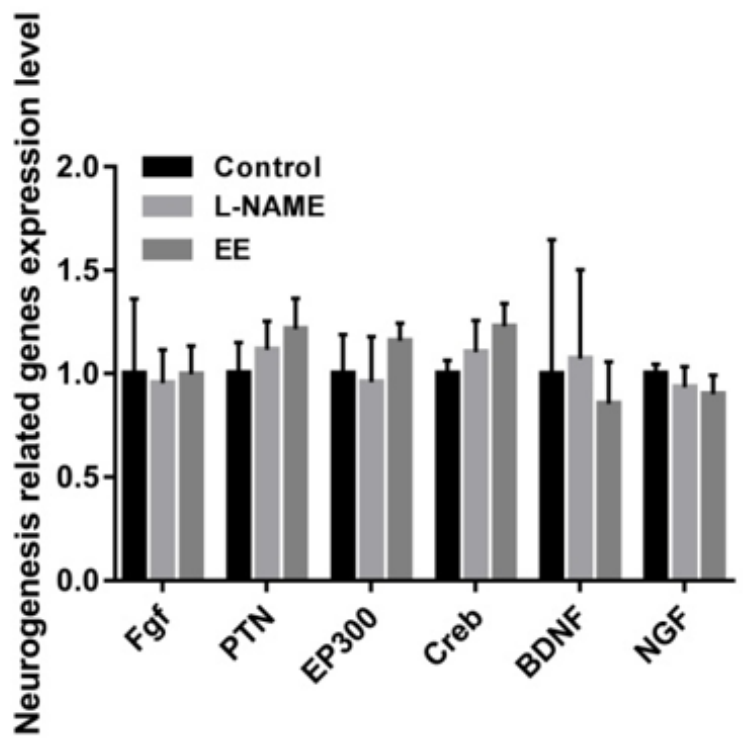

b
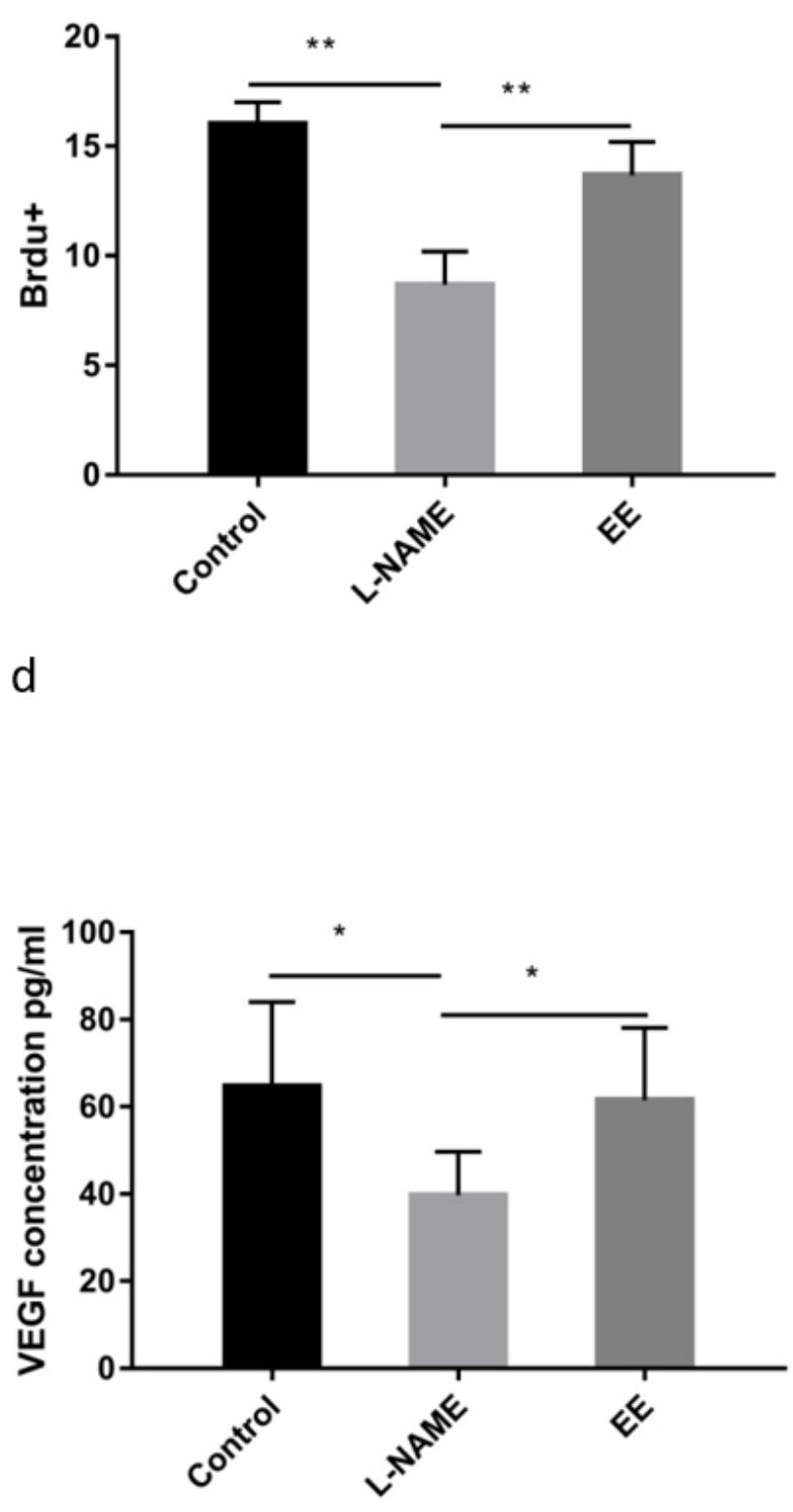

\section{Figure 5}

Analysis of progenitor cell proliferation in hippocampus of offspring. a BrdU immunofluorescence in DG section of hippocampus. b Brdu+ cells number of hippocampus of offspring in each group. $c$ mRNA levels of adult hippocampal neurogenesis-related genes. d VEGF concentration in hippocampus. Statistical analysis was performed using one-way ANOVA. * $p<0.05 . * * p<0.005$. 

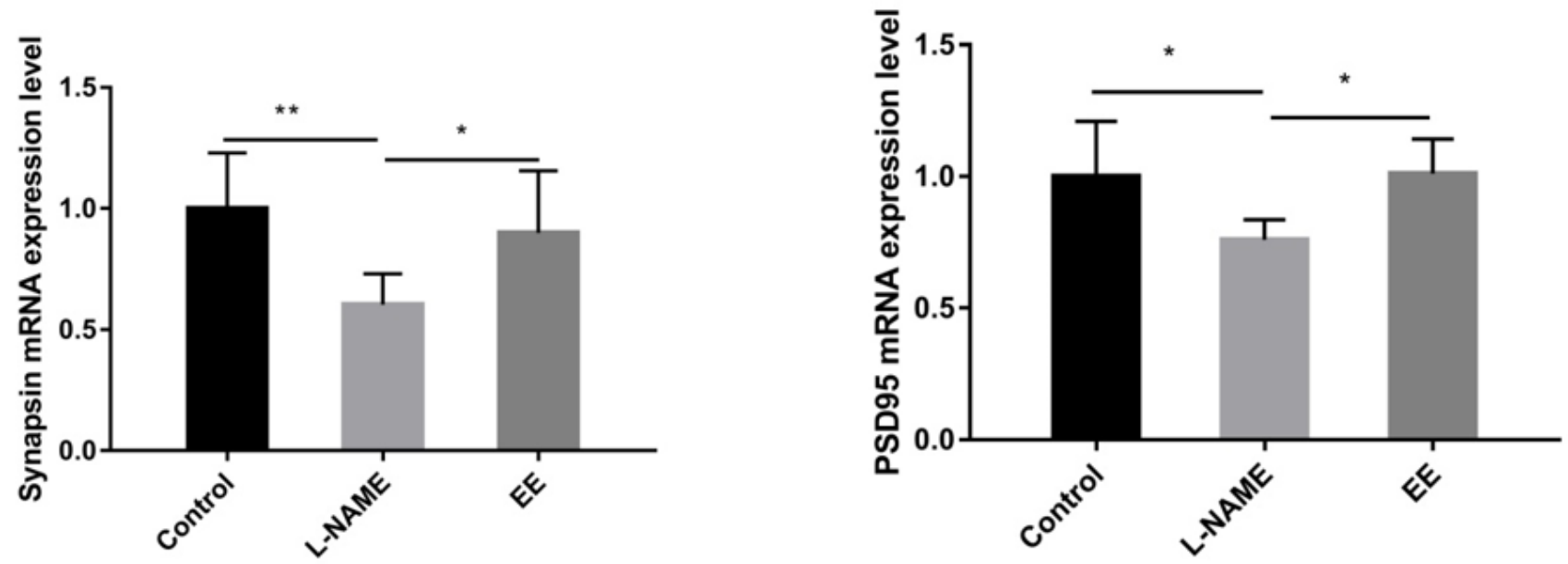

C

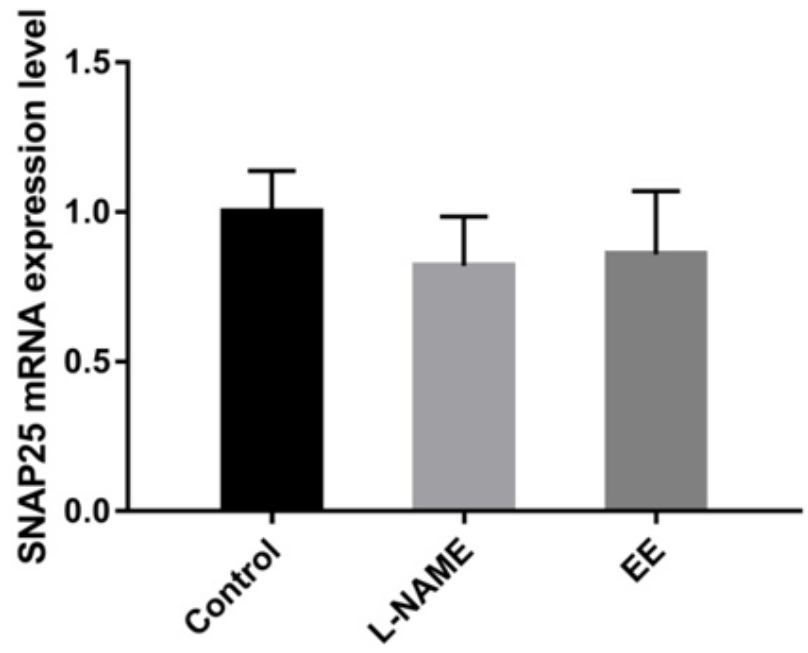

Figure 6

Analysis of mRNA expression levels of pre- and postsynaptic proteins in hippocampus of offspring. a The mRNA level of synapsin. b The mRNA level of PSD95. c The mRNA level of SNAP25. Statistical analysis was performed using one-way ANOVA. * $p<0.05 .{ }^{* \star} p<0.005$. 
a

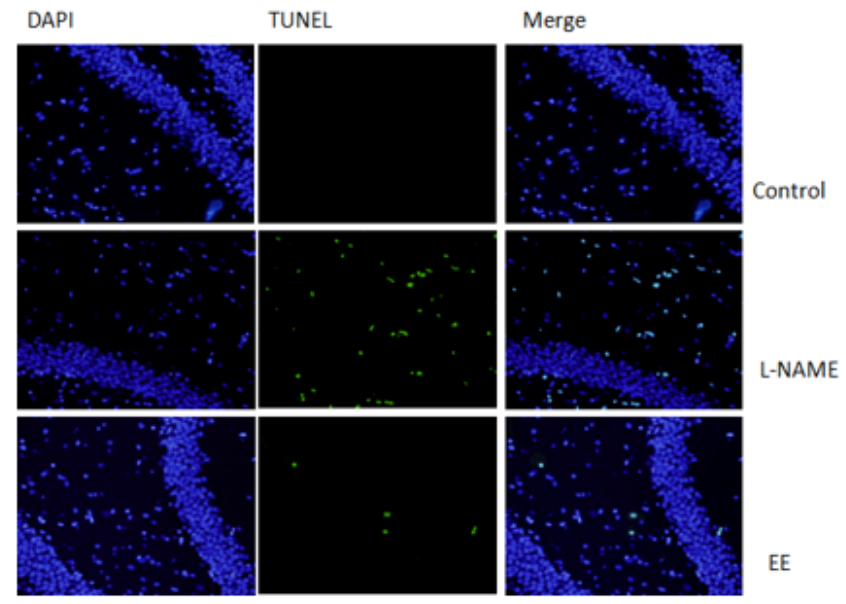

b

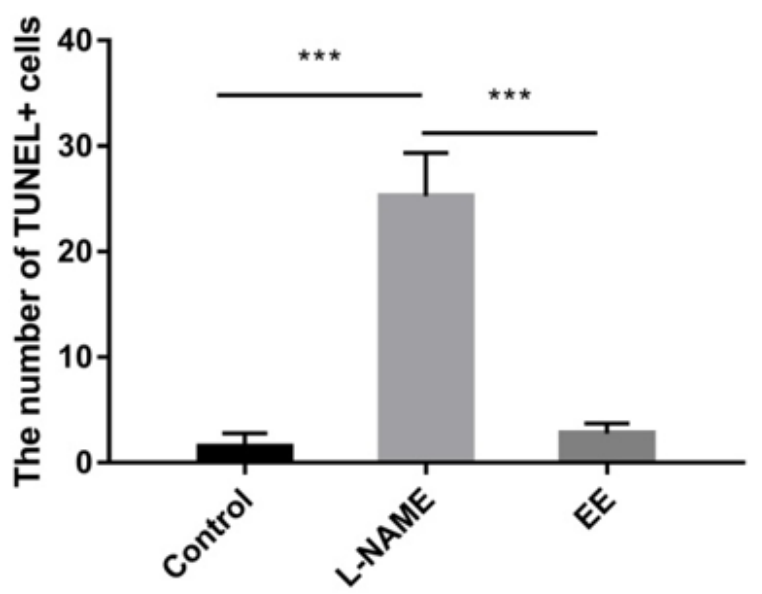

\section{Figure 7}

Analysis of neural apoptosis in hippocampus. a Tunel immunofluorescence in DG section of hippocampus. b Tunel+ cells number of hippocampus of offspring in each group. Statistical analysis was performed using one-way ANOVA. ${ }^{* \star *} p<0.001$. 

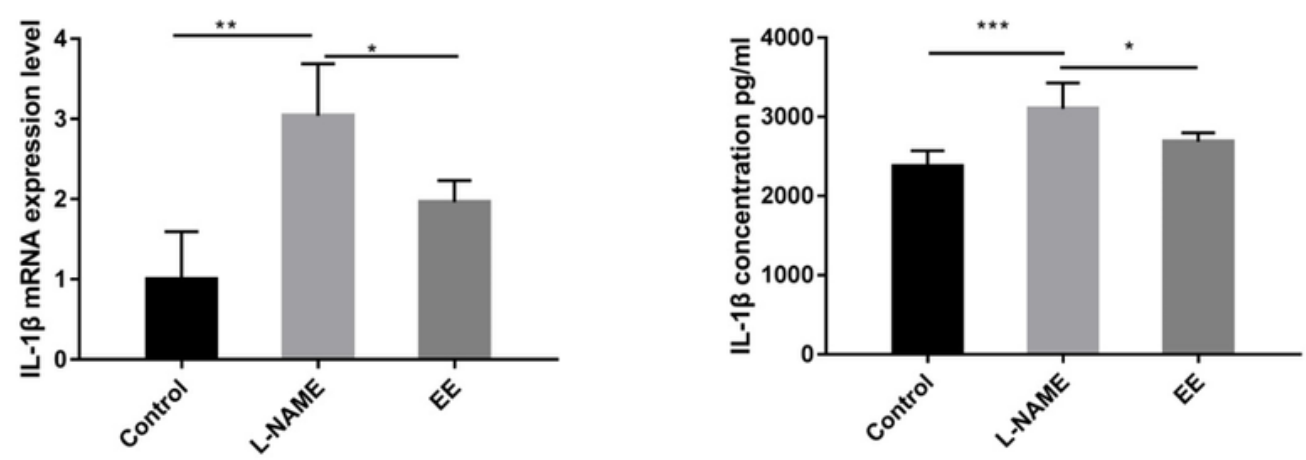

C

d
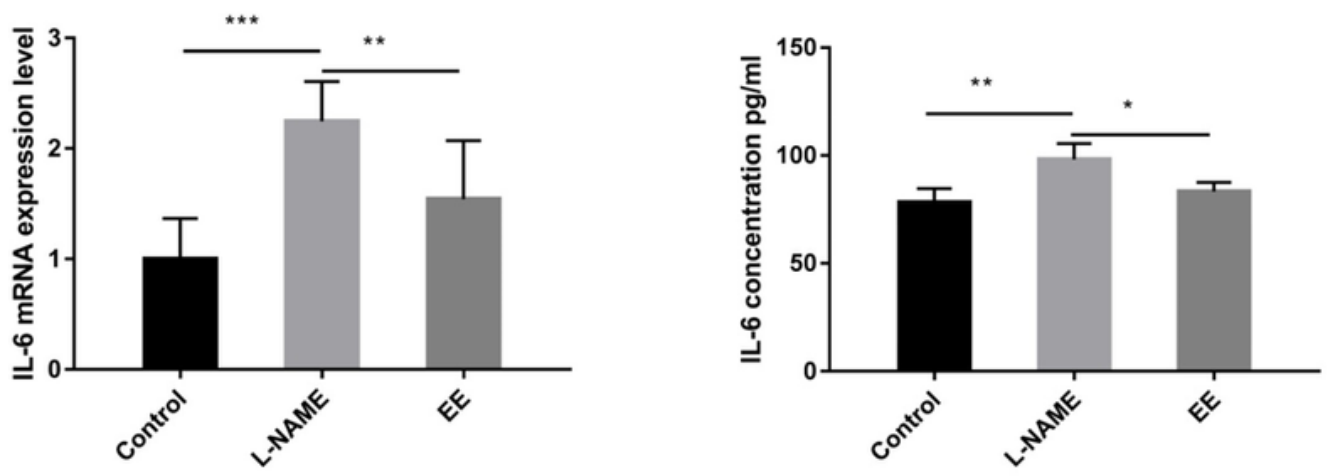

e

f
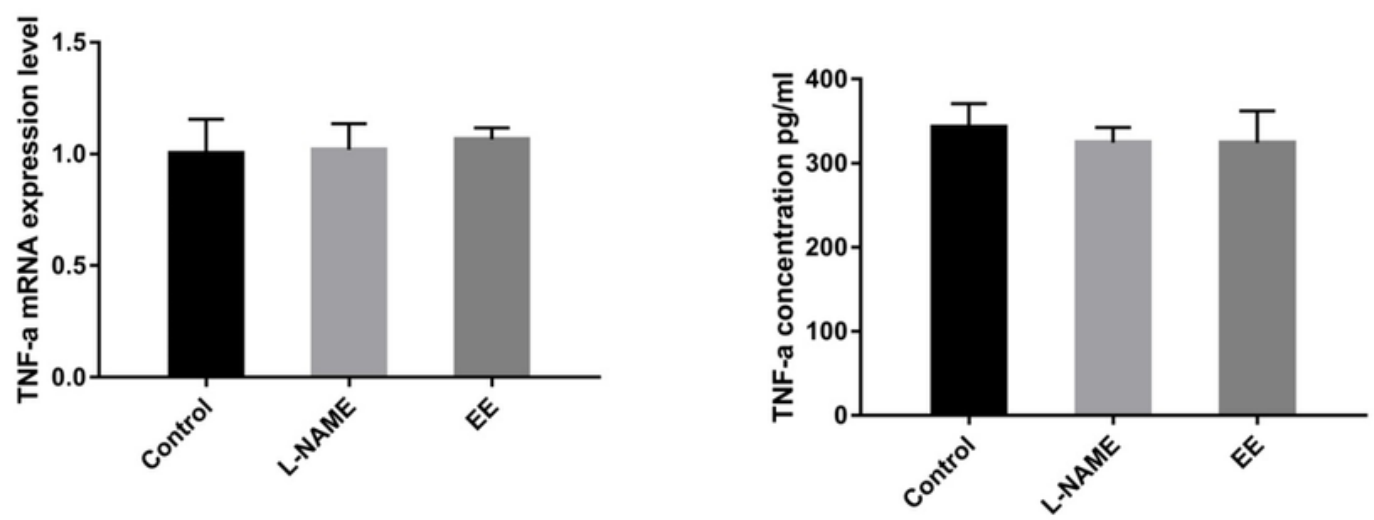

Figure 8

Effect of Environmental Enrichment on inflammatory status of hippocampus. a The mRNA level of IL-1 $\beta$ in hippocampus. $b$ The concentration of IL- $1 \beta$ in hippocampus. c The mRNA level of IL- 6 in hippocampus. $d$ The concentration of IL- 6 in hippocampus. e The mRNA level of TNF- $a$ in hippocampus. $f$ The concentration of TNF-a in hippocampus. Statistical analysis was performed using one-way ANOVA. ** $p<0.005$. ${ }^{\star \star *} p<0.001$. 